\title{
LOW-STATE MAGNETIC STRUCTURES IN POLARS: NATURE OR NURTURE?
}

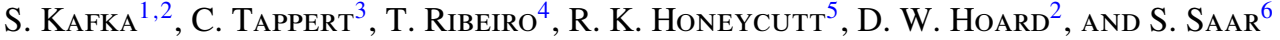 \\ ${ }^{1}$ Department of Terrestrial Magnetism, Carnegie Institution of Washington, 5241 Broad Branch Road NW, Washington, DC 20015, USA; skafka@ dtm.ciw.edu \\ ${ }^{2}$ Spitzer Science Center/Caltech, MC 220-6, 1200 East California Boulevard, Pasadena, CA 91125, USA \\ ${ }^{3}$ Departamento de Fisica y Astrofisica, Universidad de Valparaiso, Avenida Gran Bretana 1111, Valparaiso, Chile \\ ${ }^{4}$ Departamento de Fisica, Universidade Federal de Santa Catarina, Campus Trindade, 88040-900 Florianopolis, SC, Brazil \\ ${ }^{5}$ Astronomy Department, Indiana University, 319 Swain Hall West, Bloomington, IN 47405, USA \\ ${ }^{6}$ Harvard-Smithsonian Center for Astrophysics, 60 Garden Street, Cambridge, MA 02138, USA \\ Received 2010 March 8; accepted 2010 July 17; published 2010 September 14
}

\begin{abstract}
We present an orbit-resolved study of the magnetic cataclysmic variable (MCV) BL Hyi in its low state, and we explore the origin of its $\mathrm{H} \alpha$ emission line components, their properties, and their possible formation mechanism. We tentatively associate one of the line components with a high-velocity component also seen in the high state. We propose a scenario in which streaming prominence-like magnetic loops (super-prominences) are kept in place by magnetic field interactions between the white dwarf and the donor star and are responsible for the high-velocity line components in the Balmer lines. We also discuss how this is in accord with the standard scenario of the secular evolution for MCVs. Finally, we offer an observational test of our ideas and present challenges for future theoretical studies.
\end{abstract}

Key words: novae, cataclysmic variables - stars: individual (BL Hyi, AM Her, ST LMi, VV Pup, EF Eri) - stars: magnetic field

Online-only material: color figures

\section{INTRODUCTION}

Magnetic cataclysmic variables (MCVs or polars) are semidetached binary systems consisting of a late-type $(\mathrm{K} / \mathrm{M})$ dwarf and a highly magnetic white dwarf (WD; $B \geqslant 10^{7} \mathrm{MG}$ ) in an orbital period of less than $6 \mathrm{hr}$. In the "normal" accreting mode of the systems (also known as the "high" state), the M star is filling its Roche lobe, and an accretion stream which is directed toward the WD's magnetic pole(s) leaves the L1 point of the binary. Most MCVs show erratic and abrupt "low states," during which accretion through the L1 point temporarily stops, presumably due to starspots inhibiting Roche lobe overflow (Livio \& Pringle 1994). During these low states, accretion takes place through the wind of the $\mathrm{M}$ star alone at a rate of $\leqslant 10^{-12} M_{\text {sun }} \mathrm{yr}^{-1}$ (e.g., Hessman et al. 2000) and the two stellar components are exposed, allowing for a glimpse of their properties. Recent low-state spectroscopic studies of several MCVs have revealed new components of their emission lines, part of which appears to originate in magnetically controlled prominence-like features from the donor star, likely emanating from multiple locations on the star (Kafka et al. 2008). Such structures seem to be commonly encountered in low-state MCVs, but better quality of data is necessary to assess their origin, their structure, and their role in binary evolution.

In this paper, we present new spectroscopic data on the MCV BL Hyi in its low state. We analyze the structure of its emission lines and discuss the results in comparison with other MCVs. BL Hyi turns out to be the fifth member in the group of MCVs that shows complex structures in the low-state $\mathrm{H} \alpha$ emission line. We examine common properties of these group members and present a case study of the origin of the line components.

\section{BL HYI}

BL Hyi was first discovered by HEAO-1 (H0139-68) but was identified as an MCV via its photometric orbital variations and spectral characteristics (Visvanathan \& Pickles 1982). First, light curves revealed that in the high state, the system displays a hump for about two-thirds of the orbit (phases 0.00-0.65) with a steep rise and slower decay shape. Outside the hump, the brightness of the system remains constant (e.g., Thorstensen et al. 1983; Pickles \& Visvanathan 1983). Optical data revealed that the orbital period of the binary is $1.9 \mathrm{hr}$ (Pickles \& Visvanathan 1983). The strength of the main accreting pole is $23 \mathrm{MG}$, determined from Zeeman splitting of the Ly $\alpha$ line (Araujo-Betancor et al. 2005). The similarities of the orbital light curve to that of the MCV ST LMi suggest that the main accretion column is present for most of the orbit and is selfeclipsed for 0.35 phase units. X-ray and Extreme-Ultraviolet Explorer studies revealed that BL Hyi is a two-pole accretor during its "normal" accreting state (Beuermann \& Schwope 1989). According to the derived scenario, the gas forms an azimuthally extended coupling region off of the orbital plane before falling onto the main accreting pole (Mennickent et al. 1999). As in all MCVs, the magnetic field topology of the WD is not as simple as in a centered dipole. Beuermann et al. (2007) present a complex magnetic structure on the WD with magnetic field strengths between 12 and $23 \mathrm{MG}$ and faint traces of a $100 \mathrm{MG}$ field. The main accreting region was described as a "longish ribbon of field lines" reaching up to $3 R_{\mathrm{WD}}$, facing the observer at phase 0.19. Like all MCVs below the period gap $\left(P_{\text {orb }} \leqslant 2 \mathrm{hr}\right)$, BL Hyi exhibits deep low states, where the brightness of the system is reduced by $\Delta V \sim 2 \mathrm{mag}$ (from 15.3 to 17.1; Visvanathan et al. 1984). In the low state, only one pole is presumably active (Wickramasinghe et al. 1984; Araujo-Betancor et al. 2005).

\subsection{Spectroscopic Observations}

Our new spectroscopic monitoring data of BL Hyi were obtained during two nights on 2007 August 7 and 2007 August 8 UT. We used GRISM 1200R, of the FORS2 spectrograph on the ESO UT2 $8 \mathrm{~m}$ telescope, centered around the $\mathrm{H} \alpha$ line with a wavelength coverage between $6000 \AA$ and $7200 \AA$. The 


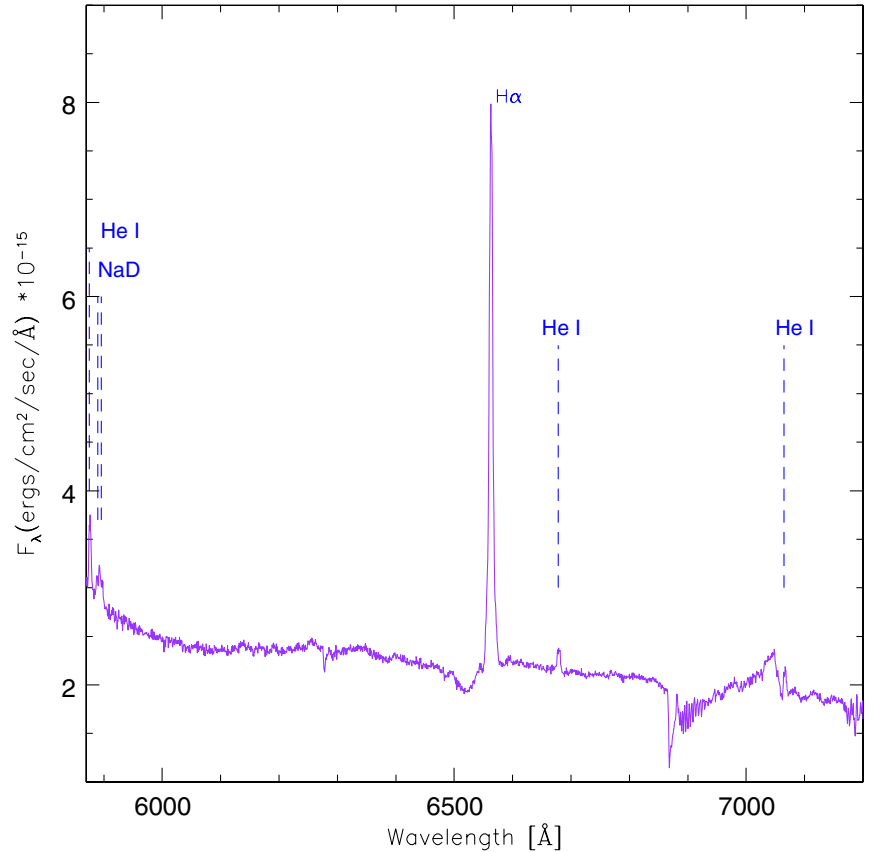

Figure 1. Averaged spectrum of BL Hyi with the emission lines marked. Note the deep blueshifted absorption trough in the $\mathrm{H} \alpha$ line due to the magnetic field of the WD (Zeeman split).

(A color version of this figure is available in the online journal.)

resulting resolution is $2.6 \AA$ (FWHM). Exposure times were mostly $400 \mathrm{~s}$ and a wavelength calibration spectrum of a $\mathrm{HeNeAr}$ lamp was obtained every 10 exposures. Taking into account comparison spectra and read-out time, a total of $\sim 70$ exposures were acquired over $\sim 4.3 \mathrm{hr}$, under clear skies. For data analysis we used the twodspec/onedspec packages of IRAF ${ }^{7}$ and custom programs (which will be discussed later).

\section{LOW-STATE EMISSION LINE CHARACTERISTICS OF BL HYI}

Figure 1 presents the average spectrum of BL Hyi with the main emission features labeled. The $\mathrm{H} \alpha$ line is in emission along with the $5876 \AA, 6678 \AA$, and $7065 \AA$ He I lines. The spectrum also presents $\mathrm{TiO}$ bands from the M5 donor star, past $7000 \AA$ in the red. The donor star is usually invisible during the normal high state; its presence in all our data confirms that BL Hyi was in the low state at the time of our spectroscopic observations (also see Wickramasinghe et al. 1984). The deep blueshifted absorption feature in the $\mathrm{H} \alpha$ line is due to Zeeman splitting from the $23 \mathrm{MG}$ magnetic field of the WD. The $\mathrm{NaD}$ lines are also in emission likely representing the irradiated inner hemisphere of the donor star, commonly present in low-state CVs.

Figure 2 displays representative spectra of BL Hyi from the two nights of our observations, centered on the $\mathrm{H} \alpha$ line. As we will see later from our analysis, the line consists of a central component and two satellites with variable visibility around the orbit, very similar to the structures seen in the Kafka et al. (2008) AM Her spectra and in all low-state polars with similar data. The spectra of the top and bottom panels, taken at similar phases but different nights, indicate that the observed features are not

\footnotetext{
7 IRAF is distributed by the National Optical Astronomy Observatory, which is operated by the Association of Universities for Research in Astronomy, Inc., under cooperative agreement with the National Science Foundation.
}

Table 1

Parameters of Radial Velocity Curve Fits of the Main Spectral Features in BL Hyi

\begin{tabular}{lcrr}
\hline \multicolumn{1}{c}{ Line } & $\gamma\left(\mathrm{km} \mathrm{s}^{-1}\right)$ & \multicolumn{1}{c}{$K\left(\mathrm{~km} \mathrm{~s}^{-1}\right)$} & \multicolumn{1}{c}{$\phi_{0}$} \\
\hline $\mathrm{H} \alpha$ & $37.4 \pm 0.8$ & $100.0 \pm 9.1$ & $-0.03 \pm 0.02$ \\
$\mathrm{He}$ I 5876 & $38.7 \pm 1.1$ & $89.7 \pm 6.5$ & $0.07 \pm 0.01$ \\
$\mathrm{NaD}$ 5889.5 & $47.1 \pm 1.7$ & $137.1 \pm 9.8$ & $0.03 \pm 0.01$ \\
NaD 5895.8 & $42.7 \pm 1.2$ & $134.9 \pm 7.2$ & $0.04 \pm 0.01$ \\
He I 6678 & $50.4 \pm 0.2$ & $117.3 \pm 5.1$ & $0.04 \pm 0.01$ \\
TiO 7051 $\AA^{\mathrm{a}}$ & $72.1 \pm 1.4$ & $221.4 \pm 10.0$ & $0.04 \pm 0.01$ \\
He I 7065 & $64.8 \pm 0.4$ & $90.6 \pm 2.8$ & $0.06 \pm 0.01$ \\
\hline
\end{tabular}

Note. ${ }^{\text {a }}$ Wavelength at the top of the bandhead.

transient (at least on timescales of several orbits). Those satellite line components seem to be absent in literature low-state spectra of BL Hyi (e.g., Beuermann \& Schwope 1989; Beuermann et al. 2007). To examine if this is due to the lower spectral resolution of the data used in previous studies or due to a (long-term) transient nature of the satellites (meaning they were not present at the times of previous observations), we used the IRAF/splot task to degrade our spectra to resolutions comparable to those in the Beuermann \& Schwope (1989) and Beuermann et al. (2007) works. In the resulting spectra the satellites indeed disappeared, confirming that higher resolution data are necessary for the detection of such structures in low-state MCVs.

Throughout this work, we use the high-state spectroscopic ephemeris ${ }^{8}$ of Mennickent et al. (1999). This ephemeris, derived from the $\mathrm{NaD}$ emission lines, defines phase zero at inferior conjunction of the donor star.

To extract further information on the origin and kinematics of the emission line components, we follow the same approach as in Kafka et al. (2008); namely, we measure line radial velocities using a single Gaussian fit for $\mathrm{He}$ I and $\mathrm{NaD}$ (which appear narrow and single-peaked) and Gaussian fit line decomposition of the $\mathrm{H} \alpha$ line profiles. Those line profiles are clearly more complex than the two or three Gaussians used, but we favor a rather conservative approach fitting the minimum number of components that reconstruct each line in order not to overinterpret our data. Using sinusoids of the form $v(t)=$ $\gamma-K \sin \left[2 \pi\left(\phi-\phi_{0}\right)\right]$ we derived the systemic velocity $(\gamma)$, the semi-amplitude velocity of the fit $(K)$ and the zero phase $\left(\phi_{0}\right)$ values for all emission line components; these are listed in Table 1. Figure 3 presents the outcome of our radial velocity (RV) measurements for the TiO bands (bandhead at 7051 the $\mathrm{NaD}$ lines, and the $\mathrm{H} \alpha$ line. Figure 4 presents the relevant measurements for the He I 5876, 6678, and $7065 \AA$ A lines. All single-peaked emission line components and the central $\mathrm{H} \alpha$ component originate on the secondary's side of center of mass.

The $K$ velocities of the $\mathrm{He}$ I and $\mathrm{NaD}$ emission lines (Table 1, see details furthermore) have values comparable to that of the $\mathrm{H} \alpha$ central component within $3 \sigma$, indicating that they originate in the same region of the system. Nevertheless, the $\gamma$ velocities of the different emission lines present distinct values that are not consistent even at the full $3 \sigma$ range. In existing studies, gamma velocities for different species have been reported to vary, but those variations have not been fully explained (e.g., Ratering et al. 1993). In BL Hyi, it is likely that these emission lines arrive from the non-uniformly irradiated face of the secondary. Therefore, these deviations can be attributed to an irradiation profile having different contributions and geometries in the different lines.

\footnotetext{
$8 \mathrm{HJD}=2450379.4725(5)+0.0789150406 \mathrm{E}$.
} 


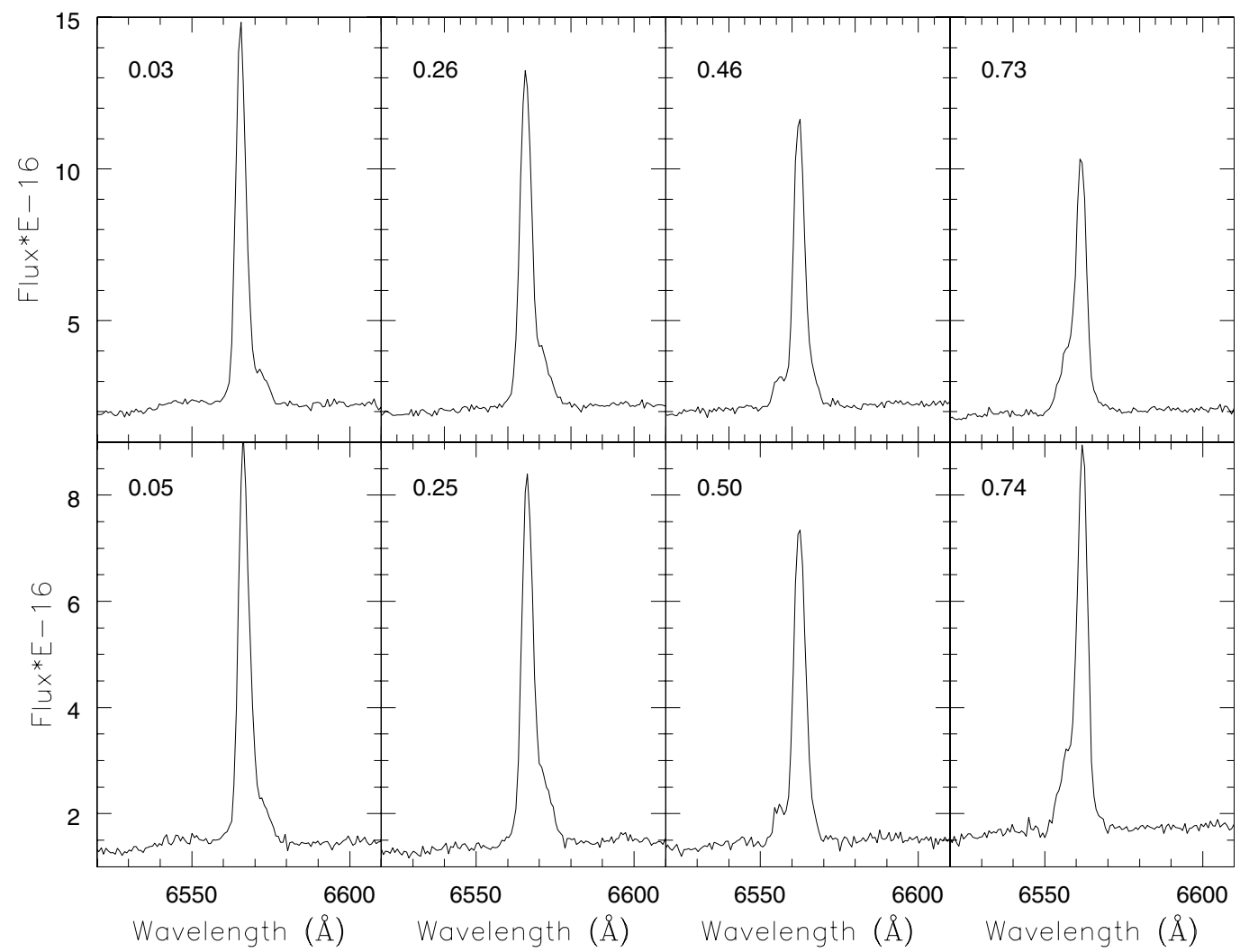

Figure 2. Snapshot sample spectra from the two nights of our observations concentrated around the $\mathrm{H} \alpha$ line. The spectra on the top panel were obtained on night 1 (2007 July 7) and the bottom on night 2 (2007 July 8). The orbital phase of each spectrum is also included. The striking similarities of the spectral line shape and components suggest that the observed satellites are not transient over several orbits in BL Hyi.

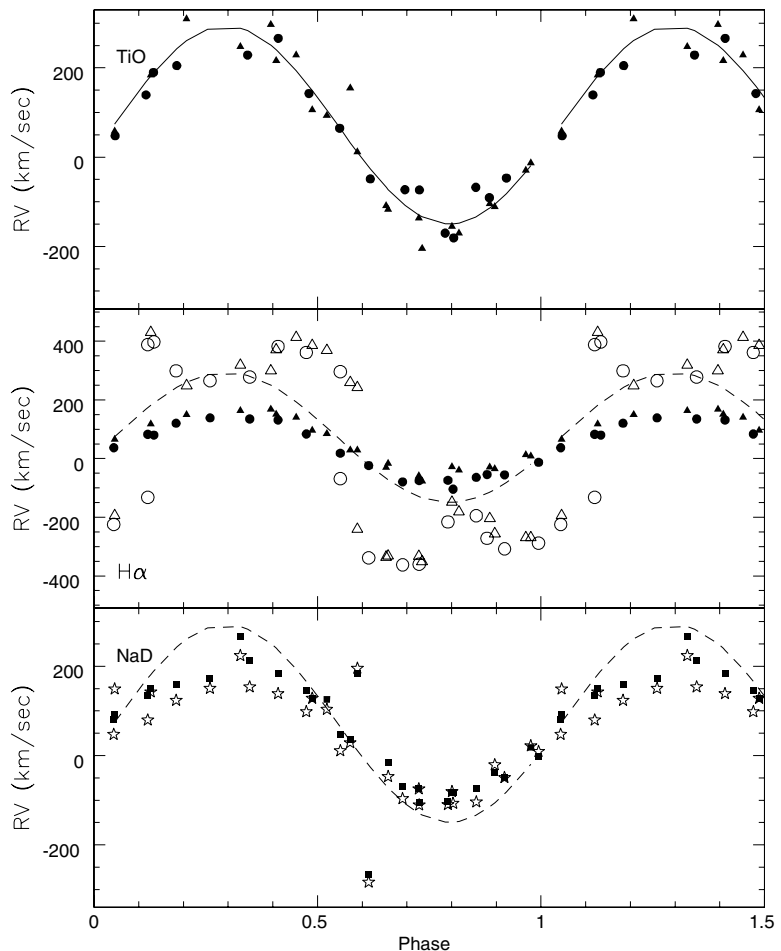

Figure 3. Radial velocities of $\mathrm{TiO}$ (bandhead at $7055 \AA$; top), $\mathrm{H} \alpha$ (middle), and $\mathrm{NaD}$ lines (bottom). In the $\mathrm{TiO}$ and $\mathrm{H} \alpha$ plots, circles are used for data of night 1 and triangles for night 2 . In the bottom panel, squares are used for the $\mathrm{NaD} 5889.51 \AA$ A line and stars for the NaD $5895.85 \AA$ line. Since TiO is usually used as a measurement of the motion (and phasing) of the center of mass of the donor star, we include the TiO RV fit in the rest of the plots, to indicate which components originate from the secondary's side of center of mass.
Figure 5 (top, left) shows the $\mathrm{H} \alpha$ line trailed spectrum of the averaged data from both nights. The lower velocity central component is easily identified with a sinusoidal behavior throughout the orbit. The satellites appear as higher velocity features bracketing the central component. Overall, the shape and appearance of the $\mathrm{H} \alpha$ trailed spectrum are strikingly similar to the appearance and phasing of the $\mathrm{H} \alpha$ line (especially the satellite components) of the AM Her low-state data (also reproduced in the bottom panel of Figure 5 and in Figure 5 of Kafka et al. 2008 for AM Her). We will discuss the nature and origin of these structures later in this paper.

In Figure 6, we present the time (top) and phased (bottom) full $\mathrm{H} \alpha$ equivalent width (EW) variability plot of the system for the two nights of our observations. In the high state, the light curve and line EWs of the system produce a hump for most of the orbits representing the direct visibility of the main accreting pole. In the low state, one emitting region is considered responsible for the (still present) pronounced hump in the soft $\mathrm{X}$-ray light curve revealing that the heated region on the WD is not negligible or absent (Beuermann \& Schwope 1989). In a similar low state, Beuermann et al. (2007) detect complex magnetic structures at the surface of the WD, with the main magnetic pole extending out to $3 R_{\mathrm{WD}}$. Our EW plot in Figure 6 shows a hint of a modulation of the $\mathrm{H} \alpha$ line during the first night of our observations, but this is absent during the second night. This is different from what was previously observed, indicating that there may have been a change in the structure of the accretion column of the system on timescales of less than a day. At the same time, the trailed spectra and Doppler maps of the system do not show any differences for the two different nights of observations, indicating that the various $\mathrm{H} \alpha$ 

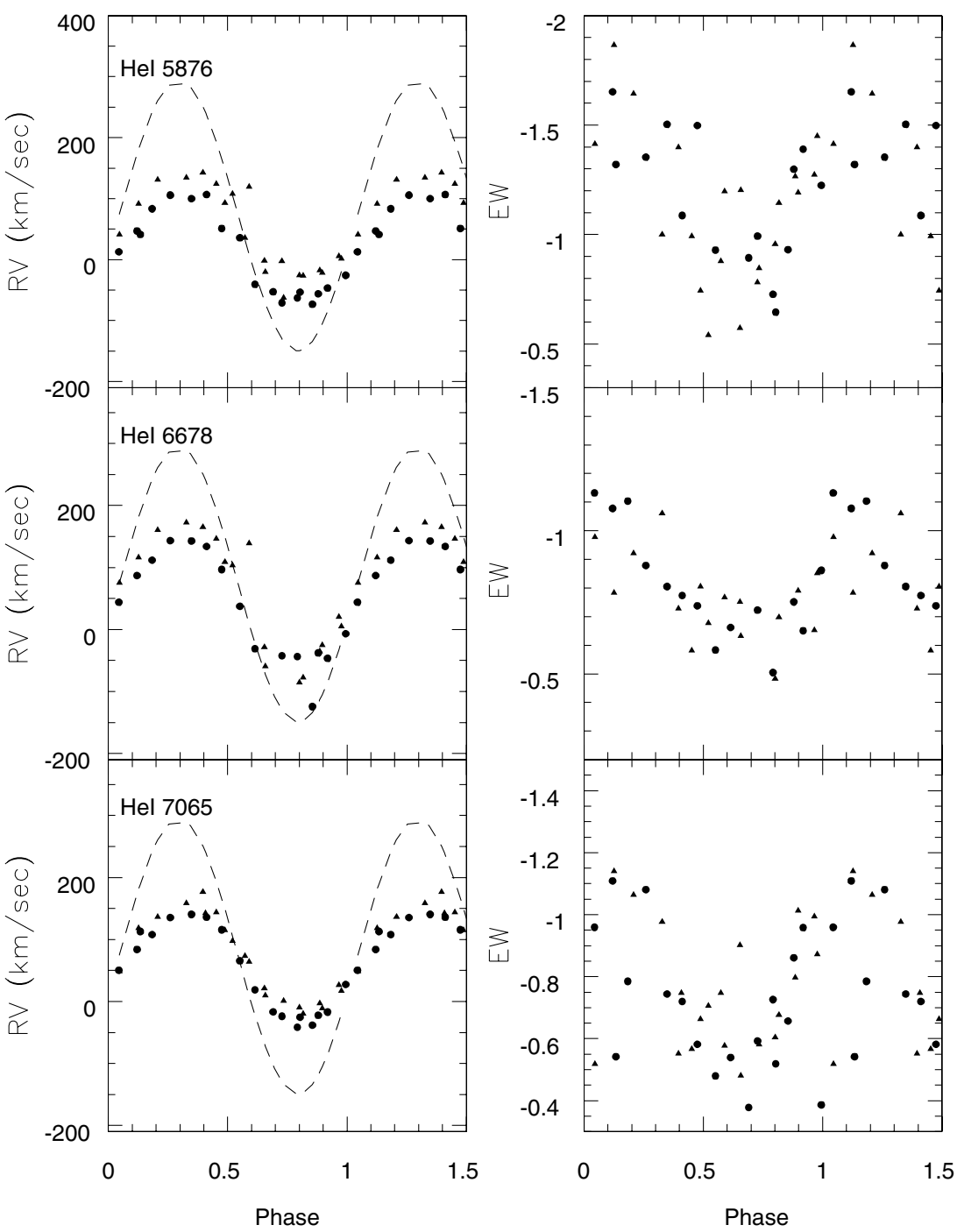

Figure 4. RVs (left) and EWs (right) of the He I lines at $5876 \AA$ (top), $6678 \AA$ (middle), and $7065 \AA$ (bottom). In the RV plots, we include the RV fit of the TiO line to demonstrate that all He I emission line components originate from near the M star in BL Hyi.

line components likely have an origin other than the high-state accretion column or accretion spot on the WD's surface. It is worth mentioning that the He I line EWs are highly modulated on both nights of our observations, which is consistent with an origin of the emission on the irradiated inner hemisphere of the M star (Figure 4).

\subsection{Doppler Maps}

Doppler tomography of $\mathrm{CV}$ is a powerful technique, providing a model for the emission line distribution in the $(\mathrm{Vx}, \mathrm{Vy})$ velocity space (e.g., Marsh \& Horne 1988; Schwope et al. 1999; Heerlein et al. 1999). In MCVs, the presence of the strongly irradiated inner hemisphere of the donor star, the ballistic/blobby accretion stream, and accretion toward one or two poles out of the orbital plane introduces additional parameters that are in need of careful modeling and understanding before they are securely identified in the $(\mathrm{Vx}, \mathrm{Vy})$-plane. Therefore, interpretation of Doppler maps should be made with caution. Nevertheless, the technique provides a tool for the identification of the kinematics of various system components; collections of MCV tomograms are abundant in the literature (e.g., Hoard 1999).

For our Doppler maps, we used the maximum entropy FORTRAN code of Spruit (1998) within an ESO-MIDAS interface. The input spectra are continuum normalized in that they are divided by a linear fit to the first and last 5 pixels of the extracted range that enters the Doppler fitting routine. The Doppler maps are computed with a smearing kernel of 2 pixels. To place the center of mass at velocity coordinates $(0,0)$, the spectra have to be corrected for the constant velocity term $\gamma$. The corresponding value from the RV fit usually serves as a first guess only and is iteratively adjusted by comparing the average original spectrum with the one reconstructed from the Doppler map. In the present case, however, the input value from the radial velocities, $\gamma=46.3 \mathrm{~km} \mathrm{~s}^{-1}$, is proved sufficiently accurate.

A standard procedure to determine what features, in the Doppler maps, are satisfying the Doppler tomography assumptions (see Marsh \& Horne 1988) is to produce maps for different half-cycles (using phases 0-0.5 and 0.5-1.0). For BL Hyi, the resulting maps for the $\mathrm{H} \alpha$ line on the first night of our observations are shown in Figure 7. The second night of data (not shown here) displays similar results, confirming that there are no significant night-to-night variations in the appearance and kinematics of the line components. Overall, the features in the two maps are quite similar, indicating that, to first order, the main assumptions of Doppler tomography are satisfied. At a very low level, though, 

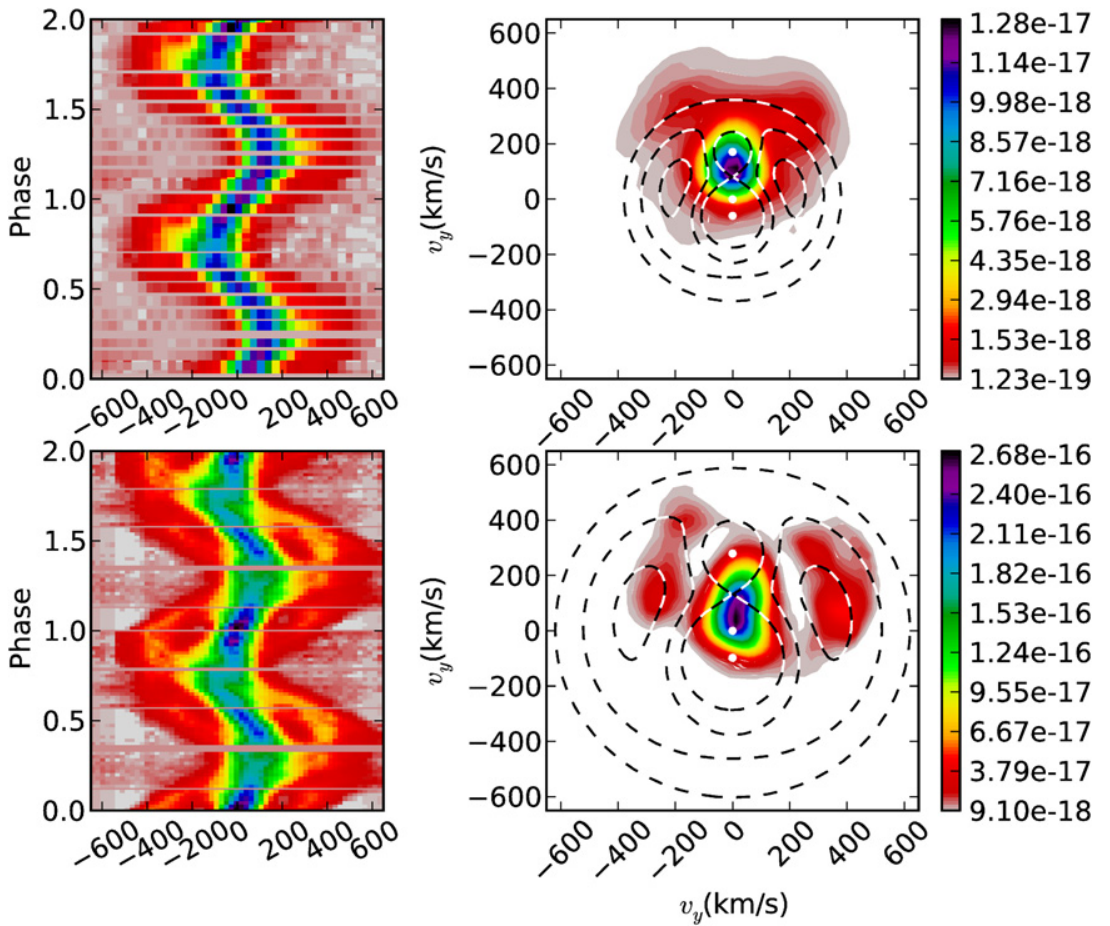

Figure 5. Trailed spectra (left) and Doppler maps (right) of BL Hyi (top) and AM Her (bottom) with the equipotential surfaces overplotted (dashed lines). Although kinematically the two bright satellites in AM Her seem to coincide with the positions of the L4 and L5 points of the binary, the Doppler map of BL Hyi does not validate this claim. See the text for discussion.

(A color version of this figure is available in the online journal.)
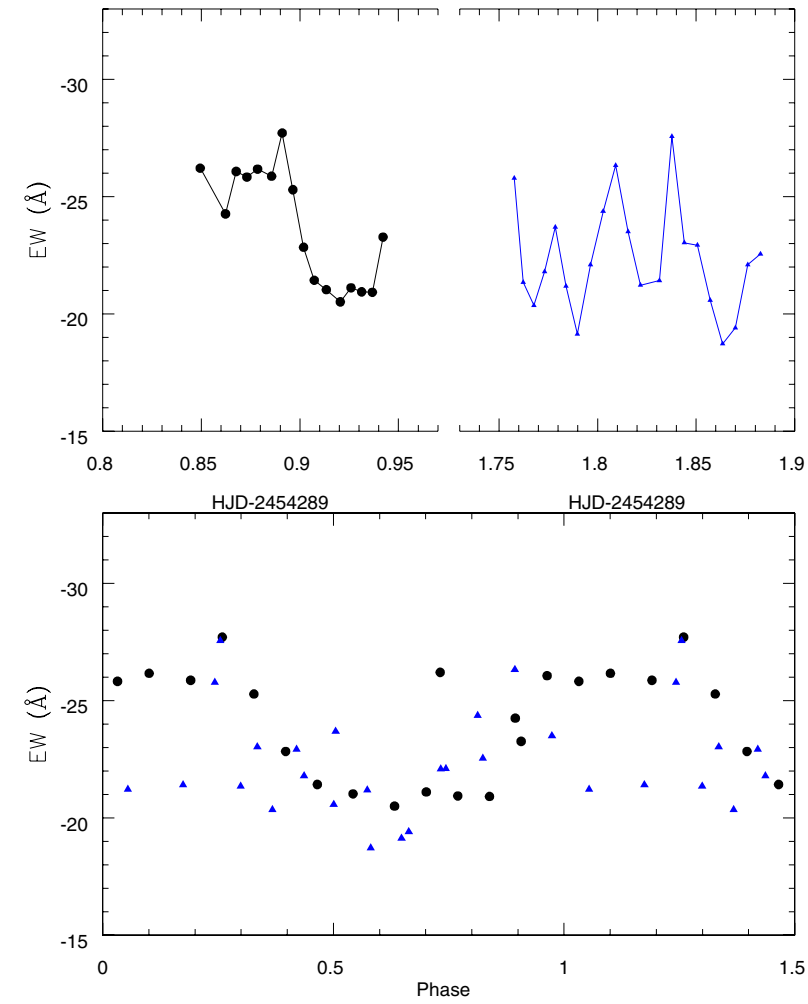

Figure 6. Time (top) and phased (bottom) light curve of the full $\mathrm{H} \alpha$ line EWs for the two nights of our observations. Although there might be a modulation of the line during the first night of our observations, it is absent during the second night.

(A color version of this figure is available in the online journal.) the satellite in the $\left(+v_{x},+v_{y}\right)$ quadrant seems to be enhanced in the second half-cycle map (Figure 7, right). This suggests that the satellites may have different $\gamma$ velocities (also supported by Table 1). This may also be evidence that the structures responsible for this satellite have a non-negligible component in the $z$-direction (Ferrario et al. 1989), which is expected for emission lines from polars (also see discussion in Heerlein et al. 1999). We will return to this point later in our discussion.

High-state Doppler maps of BL Hyi show that about 75\% of the total $\mathrm{H} \alpha$ emission line flux originates in an extended featureless low-velocity region, from the stream or from the coupling region (Mennickent et al. 1999). The accretion column and the donor star seem to be minor contributors in the emission patterns. According to the proposed scenario, the coupling region is azimuthally extended off of the orbital plane, before the accretion stream falls onto the magnetic pole. Our low-state Doppler maps of the $\mathrm{H} \alpha$ emission line of the system are shown in Figure 5 (left panel). Overplotted to the Doppler maps of Figure 3 are four equipotential surfaces, including the Roche lobe, the mass of the secondary $M_{2}=0.2 M_{\text {sun }}, q=0.3$, and $i=32^{\circ}$ (Beuermann et al. 2007). There are three prominent emission components in the low-state map: the central low velocity component, which coincides with the velocity position of the donor star of the system, being brighter around L1 and the two satellites which are present in the $\left(-v_{x},+v_{y}\right)$ and $\left(+v_{x},+v_{y}\right)$ quadrants in Figure 5. We also note the striking absence of an accretion stream in our Doppler map or any emission from the $-v_{x},-v_{y}$ quadrant, which is consistent with BL Hyi being in the low state. To accommodate our discussion, for the remainder of the paper we will refer to the $\left(-v_{x},+v_{y}\right)$ component as "satellite 1 " and to $\left(+v_{x},+v_{y}\right)$ as "satellite $2 . "$ 

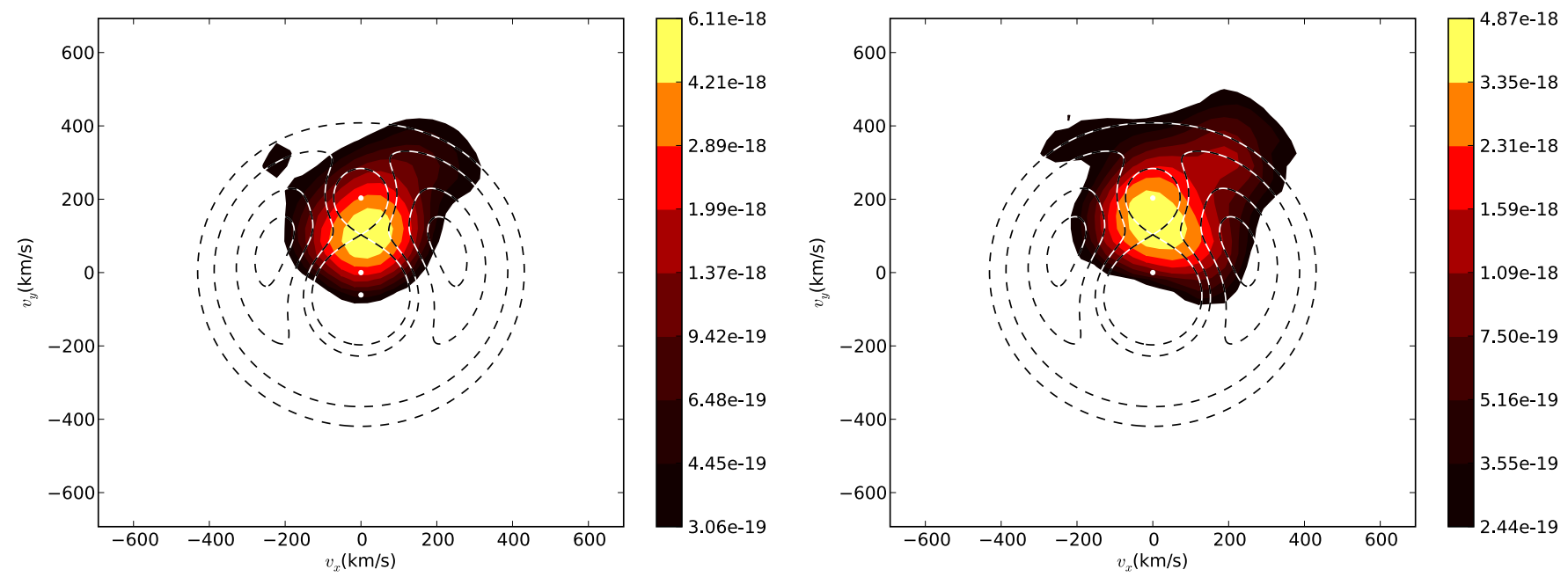

Figure 7. BL Hyi $\mathrm{H} \alpha$ emission line half-cycle Doppler maps for the night of 2007 July 7. Left: phases 0.0-0.5; right: phases 0.5-1.0. The two Doppler maps are essentially the same, suggesting that the assumptions of Doppler tomography are satisfied for the H $\alpha$ emission line in this system.

(A color version of this figure is available in the online journal.)

\subsection{Exploring System Parameters}

In AM Her, most of the emission from the central $\mathrm{H} \alpha$ component originates in a source near the center of mass of the binary, extending to the L1 point (Figure 5, bottom right panel). Kafka et al. (2008) proposed that this emission could be due to a dynamically stable magnetic "soup" which allows material to move along closed prominence-like loops emerging from the donor star, and kept in place by fast rotation and magnetic field interactions between the two stars (also see Gänsicke et al. 1998). In BL Hyi, the resulting map of Figure 5 indicates that most of the central $\mathrm{H} \alpha$ emission comes from the inner hemisphere of the $\mathrm{M}$ star (likely representing irradiation).

Considering the overall apparent similarities of the $\mathrm{H} \alpha$ line structure and kinematics between BL Hyi and AM Her, we explored the $(i, q)$ parameter space looking for a geometry in which the central $\mathrm{H} \alpha$ emission is located between L1 and the center of mass of the system; our goal was to assess whether there is any combination of $(i, q)$ with which the two satellites would coincide with the L4 and L5 points on the map. The result of this experiment is presented in Figure 8. From left to right, with dashed lines, we present the Roche geometry of the system with $i=70^{\circ}, 60^{\circ}$, and $45^{\circ}$ for mass ratios of 0.1 , 0.2 , and 0.3 (top to bottom). Perhaps the best "fit" would be the $q=0.3, i=0.60$; however, even then, the two satellites deviate significantly from the location of L4 and L5. (We will also comment on this feature later.) For the remainder of the discussion we will use the map in Figure 5, which places the main emission of the central $\mathrm{H} \alpha$ component at the $\mathrm{L} 1$ point of the system, consistent with its origin on the irradiated inner hemisphere of the $\mathrm{M}$ star.

\section{CASE STUDY OF THE SATELLITES IN THE LOW-STATE H $\alpha$ LINE IN MCVs: NATURE OR NURTURE?}

BL Hyi is the fifth MCV with satellite components in the lowstate $\mathrm{H} \alpha$ emission line. In Table 2, we present the main system characteristics of all known MCVs with $\mathrm{H} \alpha$ satellites. From this sample, the best-studied case is the prototype MCV, AM Her. More than four epochs of low-state observations over three years revealed details on the origin and nature of the emission lines of the system (Kafka et al. 2008); the emission line profiles
Table 2

MCVs with Additional Components in their $\mathrm{H} \alpha$ line

\begin{tabular}{lccccc}
\hline \hline Object & $P_{\text {orb }}$ & Donor Star & $B_{\text {WD }}$ & Inclination & $\beta^{\text {a }}$ \\
\hline & $($ hr $)$ & & $(\mathrm{MG})$ & & \\
\hline AM Her $^{\mathrm{a}}$ & 3.09 & M4-5V & 13.5 & 50 & 85 \\
ST LMi $^{\mathrm{b}}$ & 1.90 & M8V & 11.5 & 55 & 128 \\
VV Pup $^{\mathrm{c}}$ & 1.67 & M6-7V & $32 / 55$ & 75 & $145-155 / 10$ \\
EF Erid $^{\mathrm{d}}$ & 1.37 & Later than L4 & 12.6 & 55 & 75 \\
BL Hyi $^{\mathrm{e}}$ & 1.89 & M5 & 23 & 32 & 80
\end{tabular}

Notes.

a $\beta$ is the angle between the magnetic axis and the rotation axis of the WD

${ }^{\mathrm{b}}$ Kafka et al. (2008).

c Kafka et al. (2007).

d Mason et al. (2008).

e S. Kafka, unpublished data and Howell et al. (2006).

f This work.

and their characteristics seem to be similar to all systems in the table. In all systems, two "satellites" are present only in the $\mathrm{H} \alpha$ line although, in most cases, the spectral coverage of the data did not allow us to assess if they are present in the rest of the Balmer lines. ${ }^{9}$ All low-state $\mathrm{He}$ I emission lines are single-peaked and correspond to irradiation and material trapped between the center of mass of the binary and the L1 point, as is the central $\mathrm{H} \alpha$ line component.

The ambiguity of the characteristics of the $\mathrm{H} \alpha$ satellites hampers secure interpretation and determination of their origin. Specifically, in Figure 5, it is not clear if one or two sinusoids are present, whether or not they cross the central $\mathrm{H} \alpha$ component (or each other), or if they are in phase with the central component. From the different representations of our data (spectral line shapes, trailed spectra, Doppler maps) we suspect that there are likely two satellite components (in addition to the central peak of the lines); however, their phasing is critical in order to determine their origin and perhaps their formation mechanism. In BL Hyi (and in all other polars in the sample), the RV curves of the satellites can be portrayed with one or two sinusoids-crossing

\footnotetext{
9 The spectral range extends blueward to $\mathrm{H} \delta$ only in EF Eri; all Balmer lines show the same structure as the $\mathrm{H} \alpha$ line and the rest of the emission lines are single-peaked, mimicking the He I $6678 \AA$ line in amplitude and phasing.
} 


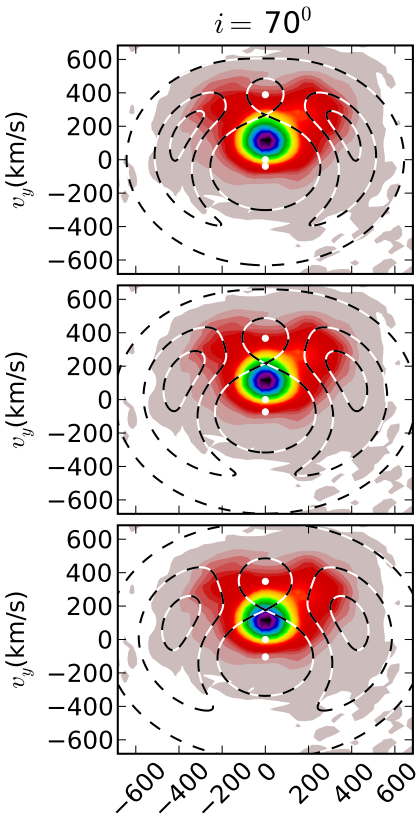

$v_{y}(\mathrm{~km} / \mathrm{s})$

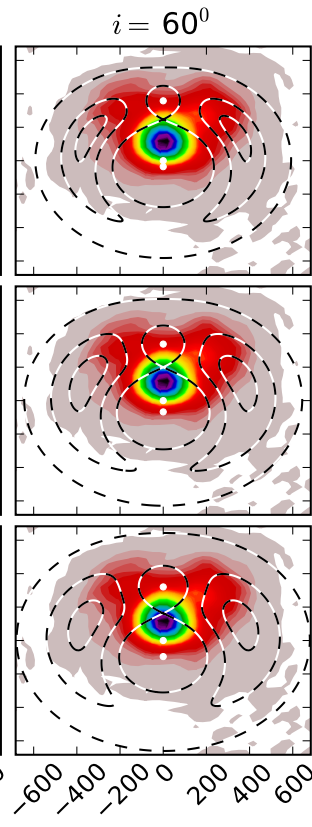

$v_{y}(\mathrm{~km} / \mathrm{s})$

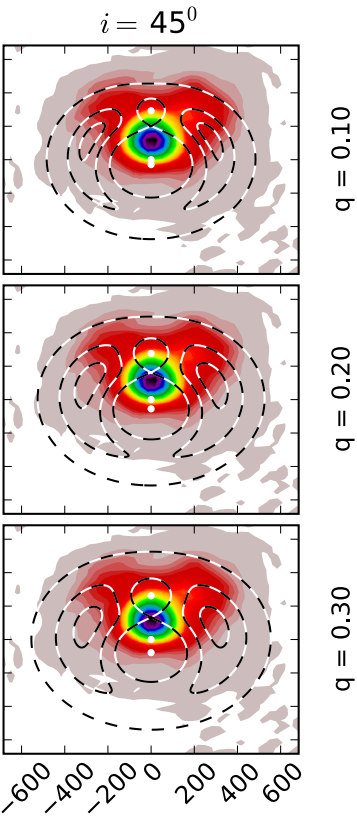

$v_{y}(\mathrm{~km} / \mathrm{s})$

Figure 8. Doppler map of Figure 5 with the equipotential surfaces overplotted for different pairs of $i$ and $q$ (dashed lines). We also assume that the central H $\alpha$ emission originates between $\mathrm{L} 1$ and the center of mass of the system. From left to right: $i=70^{\circ}, 60^{\circ}$, and $45^{\circ}$. From top to bottom, $q=0.10,0.20$, and 0.30 . This exercise concludes that there are no $(i, q)$ pairs for which the two satellite lobes coincide kinematically with the L4 and L5 of the system.

(A color version of this figure is available in the online journal.)

or not, in phase or not in phase with the central $\mathrm{H} \alpha$ line. This point needs to be resolved before we continue our discussion on their origin and physics, since our discussion and conclusions strongly depend on the properties of the satellite components that are present at any given time.

To help resolve the issue of the number and phasing of the satellite components, we created synthetic trailed spectra with all possible combinations and phasing of line components, and their relevant Doppler maps; the outcome of this exercise is presented in Figure 9.

The bottom left-side panel of Figure 9 presents two sinusoids offset by $\sim 0.05$ phase units from the central component, which crosses at phases 0.25 and 0.75 . The relevant Doppler map is strikingly similar to the Doppler maps of Figure 5, suggesting that this configuration is closer to what is observed in lowstate MCVs. The top right panel of Figure 9 presents the same satellite positioning viewed from an angle at which parts of the sinusoids are somehow eclipsed. In this case, two unconnected lobes are clearly present in the Doppler maps, positioned at the $\left(-v_{x},+v_{y}\right)$ and $\left(+v_{x},+v_{y}\right)$ quadrants of the Doppler map. Note that there is no correspondence of the location of the two lobes to the location of the L4 and L5 points in the system. The component in the $\left(-v_{x},+v_{y}\right)$ quadrant (satellite 1) is similar to the horizontal stream component of the models presented in Heerlein et al. (1999), albeit more concentrated in velocity space. It is tempting to correlate this part of the emission to material streaming toward the WD; if this is the case, then that "stream" has a smaller velocity gradient than what is observed in the accretion stream in polars. We will comment on this later in our discussion. The component in the $\left(+v_{x},+v_{y}\right)$ quadrant (satellite 2) is a new feature, never before observed in Doppler maps of MCVs. In the trailed spectrum, it corresponds to a sinusoid that leads the motion of the central $\mathrm{H} \alpha$ line component by $\sim 0.05$ phase units and has no large velocity spread over the orbit. We will implement characteristics of the line component velocities to our discussion in an upcoming session. From this exercise, though, we can conclude that the trailed spectra of the low-state MCVs (and their relevant Doppler maps) correspond to two satellites, which are crossing the sinusoid of the central component around phases 0.25 and 0.75 (quadratures of the binary) and are offset by less than 0.05 phase units from it.

In AM Her, the stability in time and phase of the satellites suggests that the related structures are stable for over three years. The existing data in VV Pup cover two weeks, during which the satellites are at the same location (Mason et al. 2008). Similarly, ST LMi was observed for about a year during which the $\mathrm{H} \alpha$ emission line has similar profiles (Kafka et al. 2007). However, this is not necessarily the case for all systems. Our spectroscopic observations of EF Eri span two years, during which those satellites are present in the $\mathrm{H} \alpha$ and the other Balmer lines ${ }^{10}$; however, later observations indicate that the structure of the $\mathrm{H} \alpha$ line has changed dramatically, showing signatures of a weak accretion stream ${ }^{11}$ (Walter 2009; S. Kafka, unpublished observations). EF Eri is the shortest period system from the collection of Table 2 and its donor star is suspected to have a sub-stellar mass (Howell et al. 2006) so it is possible that the transient nature of the satellites in EF Eri is due to its unusual donor star. Nevertheless, the fact remains that, although the $\mathrm{H} \alpha$ high-velocity satellite components seem to be a common characteristic of low-state MCVs, the relevant structures are not necessarily stable or permanent in all low-state MCVs.

\subsection{Accretion Stream?}

A question that comes to mind is whether any of the satellites are associated with any high-state emission line components in

\footnotetext{
${ }_{10}$ The spectral observations of the rest of the systems start at $5700 \AA$; thus, they do not allow one to study the properties of the satellites in other Balmer lines.

11 After our observations, EF Eri displayed intermittent short-lived "high" states which may have disrupted these structures.
} 

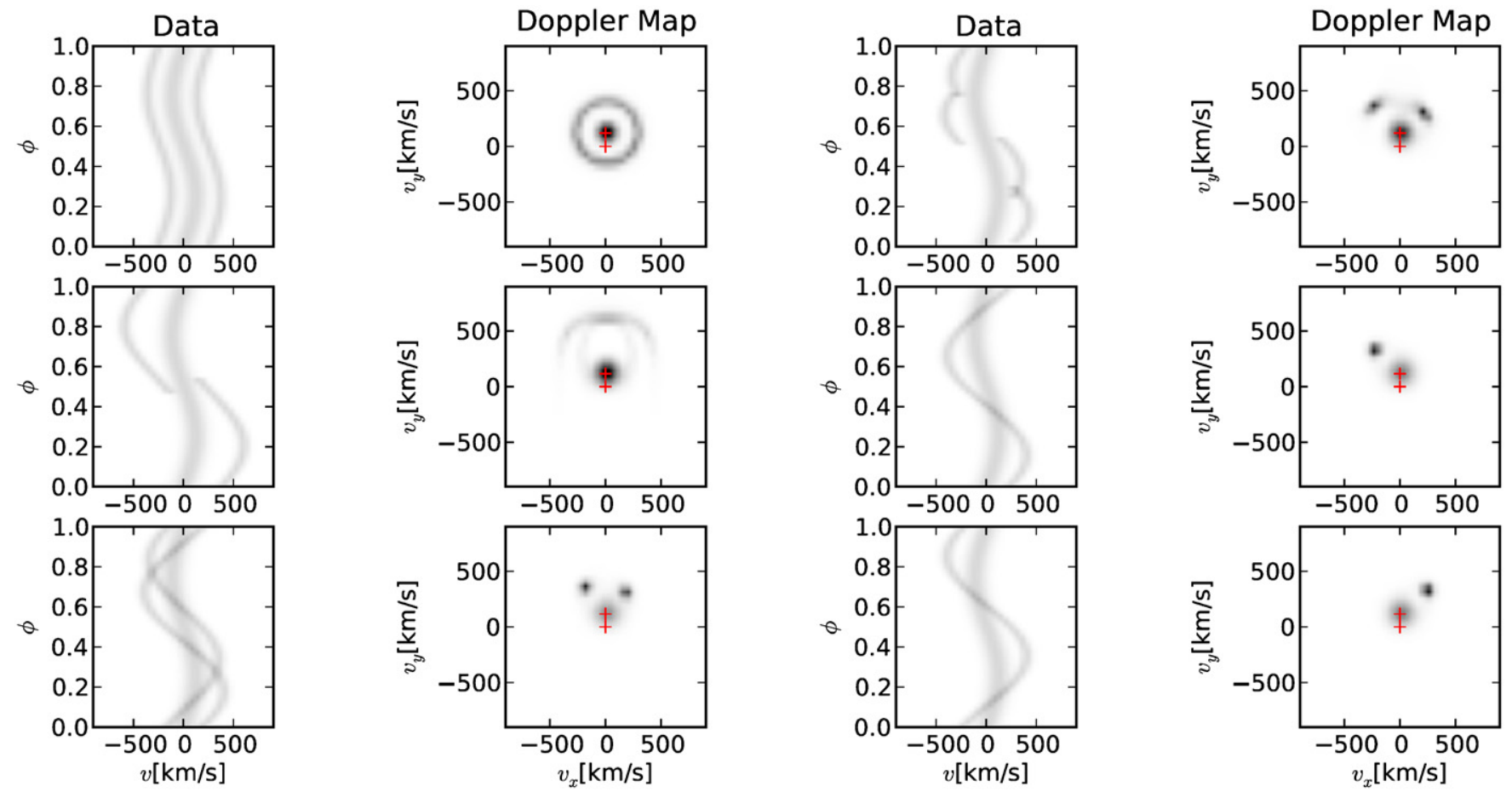

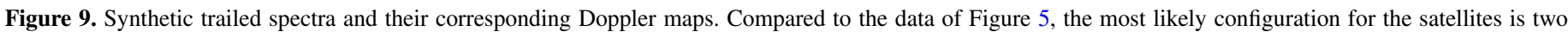
crossing sinusoids, slightly offset from the central H $\alpha$ RV curve (which corresponds to the RV curve of the inner face of the donor star).

(A color version of this figure is available in the online journal.)

MCVs. In the high state, the line profile is made of two main components, a broad one and a sharp one. These correspond to the accretion stream and the irradiated $\mathrm{M}$ star, respectively. ${ }^{12}$ It is tempting to associate one or two of the low-state satellite components as being a remnant of the wide high-state component of the systems (which is related to the accretion stream). Here, we review the main high-state accretion characteristics of the CVs of Table 2; they are also summarized in the table. An extended discussion of BL Hyi is presented in Section 1 and will not be repeated here.

AM Her. The high-state Doppler map of AM Her consists of a "focused and well-defined accretion stream" and a component originating from the asymmetrically irradiated inner hemisphere of the M star (Watson et al. 2003). The primary magnetic pole leads the $\mathrm{M}$ dwarf by $12^{\circ}$. Soft X-rays are generated from the primary accreting pole between orbital phases 0.3 and 1.1, whereas a secondary pole contributes to cyclotron emission between phases 0.7 and 1.15 (Ramsay et al. 1996).

ST LMi. Robertson et al. (2008) present echelle spectra of the emission lines of ST LMi in the high state. The magnetic pole of the system leads the M star by $42^{\circ}$ and lies at co-latitude of $141^{\circ}$ on the WD. The light curve of the system is similar to BL Hyi, presenting a "hump" when the main accreting pole is visible between orbital phases 0.55 and 0.90 . The light curve is flat outside those phases, when the accreting pole is self-eclipsed by the WD. The emission lines consist of two components; a broad component coinciding with the accretion stream infalling on the magnetic pole of the WD and a narrow component from the M-dwarf's side of the center of mass, offset by $\sim 0.25$ phase units from the M-dwarf RV (defined in Kafka et al. 2007). This narrow component was attributed to the location where the magnetic fields of the two stars interact.

12 The emission lines can have up to four components (Mukai 1988).
VV Pup. The optical light curve of the system is similar to BL Hyi and ST LMi-it consists of a single hump, visible between phases 0.7 and 1.15, and it is self-eclipsed outside that (Ramsay et al. 1996). This system has also been reported to be a two-pole polar in the high state (Wolff et al. 1999). Diaz \& Steiner (1994) discuss the structure of the high-state $\mathrm{H} \alpha$ line components, attributing $40 \%$ to the stream, $30 \%$ to the irradiated inner hemisphere of the M star, and 30\% to the coupling region/ accretion column.

EF Eri. In the high state, the system is a two-pole accretor, with the two opposite-polarity spots on the same hemisphere, separated by $77^{\circ}$ in latitude (Oestreicher et al. 1990). Beuermann et al. (2007) present a comprehensive map of the magnetic properties of the WD of EF Eri, concluding that the magnetic field configuration is not a simple dipole (it is rather a multipole) with the main magnetic axis offset by $30^{\circ}$ from the rotational axis of the WD. The main accretion spot is mostly facing the observer at phase 0.10 .

In all five polars, a comparison of the phasing and properties of their high-state accretion stream and Doppler maps reflected in their $\mathrm{H} \alpha$ line reveals striking differences with their properties in the low-state $\mathrm{H} \alpha$ line. However, in the high-state AM Her spectra, the weak high-state, high-velocity component of the Si IV line (HVC-Si IV) reported in Gänsicke et al. (1998) could be associated with satellite 1 . This HVC-Si v line component seems to be a little offset in phase with respect to the central Si IV component (the authors note that it is not strictly sinusoidal in shape) and it is overwhelmed by light from the main accretion stream itself. HVC-Si v does not make a significant contribution in the relevant high-state Doppler maps and it is attributed to a part of the accretion stream closer to the WD. If satellite 1 represents the HVC-Si IV from an accretion stream, then material should be escaping through L1 even during the low states. The current scenario for the CV low states supports that they are due to starspots on the secondary covering 
L1 (Honeycutt \& Kafka 2004) and interrupting the accretion stream. Hessman et al. (2000) present a starspot filling factor of $\sim 50 \%$ of the donor star's surface around L1 for AM Her, indicating that this L1 starspot coverage could not be completely blocking material from escaping and flowing toward the WD even at a much lower rate than the high-state accretion stream.

Satellite 2 is a new feature, unique in the low state (or high state) of the systems-it is not present in any of the high-state Doppler maps and cannot be associated with any of the highstate emission line components. This component is present in all systems in the sample, at the same location in each system, regardless of the differences in the magnetic field geometry of each polar and their high-state accretion characteristics. Therefore, its origin needs to be sought elsewhere.

\subsection{Magnetic Field Interactions or Stellar Activity?}

The systems in Table 2 comprise a very diverse group of stars with quite different properties; the members of the group have a variety of orbital periods, WD magnetic field strengths, accretion properties (one or two pole accretors), and masses of the donor star. The only common property of all systems is the magnetic nature of their WD and the presence of the two $\mathrm{H} \alpha$ satellites in their low states at about the same orbital phases (i.e., their location in the binary) with respect to the central component. In our previous discussion, we suggested that satellite 1 could be part of the high-velocity accretion stream, also identified with the high-state HVC-Si IV of Gänsicke et al. (1998). Even if this is the case, we still need to understand the properties (and origin) of satellite 2 .

In the Doppler map, both satellites seem to have a smaller spread in their $v_{x}$ and $v_{y}$ velocities, whereas an L1-overflow accretion stream increases its $v_{x}$ as it gets further away from the L1 point. Looking at the Doppler map of AM Her in Figure 5, the high-velocity emission region could be interpreted as originating on the L4 and L5 Lagrangian points of the binary (which was also pointed out in Kafka et al. 2008); however, this is not the case for BL Hyi and for the rest of the MCVs. The L4 and L5 points in a binary are stable dynamical points for $M_{1} / M_{2} \geqslant$ 24.96 , but in the case of the systems in Table 2, this condition can be satisfied only for impossibly large WD masses $\left(M_{1} \geqslant 2 M_{\odot}\right)$. Therefore, dynamically, the L4 and L5 points are unlikely hosts of the material responsible for the satellite emission in polars.

In Kafka et al. (2005a, 2007, 2008), those satellites have been attributed to material magnetically trapped in large superprominence loops arising from the donor star and they likely have a more complex structure. The geometry (and visibility) of the satellites suggest that those prominences could be around the equator of the M star (or along the orbital plane). Similarly, in single M dwarfs, "slingshot prominences" 13 are confined close to the equatorial plane of the star and have a projected area along the direction of the observer several percent of the total stellar surface area. Theoretical calculations of dipole, quadrupole, and sextapole fields in single stars demonstrate several equilibrium points above the stellar surface, where material can linger. A necessary and sufficient condition for their presence is for the mass density per unit flux to be almost stable (or to decrease slowly) in the direction of the effective gravity of the star (Ferreira 2000). A stable equilibrium can thus be retained for a few hundred Gauss of magnetic field strength; this is observed

\footnotetext{
13 Slingshot prominences in fast-rotating single stars correspond to material accumulated at a height where centrifugal forces balance gravity and magnetic tension in a corotating atmosphere (Collier Cameron \& Robinson 1989).
}

in fast-rotating low-mass stars and is definitely expected in MCV donor stars. Slingshot prominences have been previously reported to be present in disk CVs above the period gap, but their lifetimes seem to be of the order of a few days (e.g., Watson et al. 2007). In single stars, circumstellar loops of magnetically confined material are longer lived, but their prominences evolve on timescales of hours (Donati et al. 2000; Dunstone et al. 2006). In polar donor stars, we have indications that these satellites are persistent and stable and have the same structure on timescales of years (e.g., AM Her; Kafka et al. 2008). It is very likely that in polars, magnetic field interactions between the WD and its companion could provide a stabilizing mechanism.

In this regard, the answer to the puzzle probably lies in the geometry and configuration of the magnetic field interactions between the two stars in the binary. A series of papers (Li et al. 1994, 1995; Li \& Wickramasinghe 1998) exploring the principles and geometry of magnetic braking in polars introduce the concepts of areas of open magnetic field lines ("wind zones") and areas of closed magnetic field lines ("dead zones") in MCVs. Our special interest in this configuration is the location of the system's dead zones, which are closed magnetic field loops connecting the two stars. In the dead zones, the magnetic pressure is greater than the thermal pressure of the gas, enabling gas flow from the M star to the WD, leading to angular momentum exchange (Li \& Wickramasinghe 1998). The magnetic dipole orientation of the two stars with respect to each other and with respect to the orbital plane is a key factor in the formation of the dead zones in the binary. The authors conclude that, if the inclination of the WD magnetic dipole is less than $25^{\circ}$ in $\mathrm{MCVs}$, the dead zones are more efficient, leading to a reduction of open magnetic field lines, which are in turn responsible for magnetic braking. This explanation is consistent with magnetic braking being less important in MCVs (which is observationally confirmed). This also provides numerous channels for material exchange between the two stars. Material can flow within the dead zones of the binary, with little (or no) changes in its velocity since it is confined by the magnetic field of the binary. This is the principle of the "magnetic siphon" that is considered to be in action in detached magnetic WD-M dwarf binaries (Webbink \& Wickramasinghe 2005). However, Kafka et al. (2010) recently reported spectroscopic observations of the detached short-period $(P \sim 4.2 \mathrm{hr}$ ) magnetic WD/dMe binary J204827.9+005008.9, in which such satellites are not present, even though the magnetic WD is accreting the wind of its companion at a rate similar to that in low-state polars, ruling out that the satellites are due to a magnetic siphon alone. This is also an indication that these satellites are somehow connected to the accretion history of the binary (perhaps they are formed when the donor star first comes in to contact with its Roche lobe), and thus they are expected to be present in semi-detached systems alone.

In the polars of our sample, the key ingredient in the magnetic confinement scenario is that the $\mathrm{M}$ star needs to have a largescale dipole field (which is also the means for magnetic braking leading to angular momentum loss from the binary). Recent observations of M4 stars revealed the presence of axisymmetric large-scale poloidal fields (Morin et al. 2008); their origin is still unknown. At the same time, this allows for large-scale dipole structures to be possible in polar donor stars, even in the case of fully convective M dwarfs. Therefore, if those satellite components are somehow formed as a result of the accretion process, then they could be a stable feature of the system from the very beginning of its formation as a polar. According to the 
standard scenario for $\mathrm{CV}$ evolution, $\mathrm{CV}$ s form when the $\mathrm{M}$ star comes into contact with its Roche lobe which, in most cases, happens when the binary is above the period gap (and the donor star is not fully convective). The binary evolves toward shorter periods by losing mass and angular momentum from magnetic braking. If those satellite structures are somehow associated with the process of the $\mathrm{M}$ star coming into contact with its Roche lobe, then as long as the $\mathrm{M}$ star remains in contact with its Roche lobe, the satellites will always be present in the binary, regardless of the secular/structural evolution of the donor star.

Observational evidence indicates that there is no significant period gap for MCVs (Webbink \& Wickramasinghe 2002). This means that the donor stars in MCVs never detach from their Roche lobes once the $\mathrm{CV}$ is formed and the flow of material from the magnetic structures that are represented by the satellite structures is never interrupted during the life of the CV. Therefore, they can be present even when these donor stars are fully convective, which is the case in most systems in Table 2. In this scenario, at least one of the satellites (satellite 2) represents material flowing along super-prominences (large magnetic loops) formed in the dead zones of the binary, either connecting the two stars or on the M star itself.

\section{FINAL REMARKS}

We present an orbit-resolved study of the MCV BL Hyi in its low state and explore the origin of its $\mathrm{H} \alpha$ line components. We conclude that the characteristics of those satellites are similar to those found in earlier studies of other low-state MCVs. We also present an overview of the properties of the satellites for all the group members outlining their properties and attempting to pinpoint their origin. Our main findings are listed below.

1. We report the presence of two satellites' features in $\mathrm{H} \alpha$ in BL Hyi which have a sinusoidal-like appearance in the trailed spectrum and cross the sinusoid of the central $\mathrm{H} \alpha$ line component. They also have a phase shift with respect to the central component.

2. Dynamical arguments and our Doppler maps support that this material cannot originate from the L4/L5 points of the binary, since this would require impossibly large M-dwarf masses.

3. In the five polars we compared, the phasing and appearance of the low-state satellite 1 line component are similar to that of the high-state AM Her feature identified as HVC-Si IV.

4. Satellite 2 likely has a magnetic origin in the form of large loop prominence-like material released from the active donor star. Magnetic field interactions between the two stellar components should keep those structures in place for times longer than the observed lifetimes of prominences in single fast rotating stars. This emission can be stationary and/or can be streaming toward the WD; the kinematics are uncertain reflecting the lack of relevant theoretical work.

5. Our comparison with the emission line properties of $\mathbf{J} 2048$ (detached magnetic WD-M dwarf) indicates that these super-prominences are not likely to result from magnetic field interactions between the magnetic WD and its companion alone. Their formation mechanism and their role on the evolution of the binary is a big puzzle, providing a challenging problem for theoretical models. Most importantly, we do not know the association of those features with the accretion process and their importance for the secular evolution of $\mathrm{CVs}$.
Since the emitted gas in those satellites is formed because of material ejected in prominence-like structures, they are likely present in disk CVs above the period gap as well, however, their evolution with time is unknown since, in that case, there is no strong WD magnetic field to ensure their longevity and stability.

\section{REFERENCES}

Araujo-Betancor, S., Gänsicke, B. T., Long, K. S., Beuermann, K., de Martino, D., Sion, E. M., \& Szkody, P. 2005, ApJ, 622, 589

Beasley, A. J., \& Guumldel, M. 2000, ApJ, 529, 961

Beuermann, K., Euchner, F., Reinsch, K., Jordan, S., \& Gänsicke, B. T. 2007, A\&A, 463, 647

Beuermann, K., \& Schwope, A. D. 1989, A\&A, 223, 179

Bonnet-Bidaud, J. M., et al. 2000, A\&A, 354, 1003

Collier Cameron, A., \& Robinson, R. D. 1989, MNRAS, 238, 657

Cropper, M. 1987, MNRAS, 228, 389

de Martino, D., et al. 1998, A\&A, 333, L31

Diaz, M. P., \& Steiner, J. E. 1994, A\&A, 283, 508

Donati, J.-F., Mengel, M., Carter, B. D., Marsden, S., Collier Cameron, A., \& Wichmann, R. 2000, MNRAS, 316, 699

Dunstone, N. J., Cameron, A. C., Barnes, J. R., \& Jardine, M. 2006, MNRAS, 373, 1308

Ferrario, L., Tuohy, I. R., \& Wickramasinghe, D. T. 1989, ApJ, 341, 327

Ferreira, J. M. 2000, MNRAS, 316, 647

Gänsicke, B. T., Fischer, A., Silvotti, R., \& de Martino, D. 2001, A\&A, 372, 557

Gänsicke, B. T., Hoard, D. W., Beuermann, K., Sion, E. M., \& Szkody, P. 1998, A\&A, 338, 933

Heerlein, C., Horne, K., \& Schwope, A. D. 1999, MNRAS, 304, 145

Hessman, F. V., Gänsicke, B. T., \& Mattei, J. A. 2000, A\&A, 361, 952

Hoard, D. W. 1999, in ASP Conf. Ser. 157, Annapolis Workshop on Magnetic Cataclysmic Variables, ed. C. Hellier \& K. Mukai (San Francisco, CA: ASP), 201

Honeycutt, R. K., \& Kafka, S. 2004, AJ, 128, 1279

Howell, S. B., Harrison, T. E., Campbell, R. K., Cordova, F. A., \& Szkody, P. 2006, AJ, 131, 2216

Howell, S. B., Walter, F. M., Harrison, T. E., Huber, M. E., Becker, R. H., \& White, R. L. 2006, ApJ, 652, 709

Kafka, S., Honeycutt, R. K., Howell, S. B., \& Harrison, T. E. 2005a, AJ, 130, 2852

Kafka, S., Howell, S. B., Honeycutt, R. K., \& Robertson, J. W. 2007, AJ, 133, 1645

Kafka, S., Ribeiro, T., Baptista, R., Honeycutt, R. K., \& Robertson, J. W. 2008, ApJ, 688, 1302

Kafka, S., Robertson, J., Honeycutt, R. K., \& Howell, S. B. 2005b, AJ, 129, 2411

Kafka, S., Tappert, C., \& Honeycutt, R. K. 2010, MNRAS, 403, 755

Li, J., \& Wickramasinghe, D. T. 1998, MNRAS, 300, 718

Li, J., Wickramasinghe, D. T., \& Wu, K. 1995, MNRAS, 276, 255

Li, J. K., Wu, K. W., \& Wickramasinghe, D. T. 1994, MNRAS, 268, 61

Livio, M., \& Pringle, J. E. 1994, ApJ, 427, 956

Marsh, T. R., \& Horne, K. 1988, MNRAS, 235, 269

Mason, E., Howell, S. B., Barman, T., Szkody, P., \& Wickramasinghe, D. 2008, A\&A, 490, 279

Mennickent, R. E., Diaz, M. P., \& Arenas, J. 1999, A\&A, 352, 167

Morin, J., et al. 2008, MNRAS, 390, 567

Mukai, K. 1988, MNRAS, 232, 175

Oestreicher, R., Seifert, W., Wunner, G., \& Ruder, H. 1990, ApJ, 350, 324

Pickles, A. J., \& Visvanathan, N. 1983, MNRAS, 204, 463

Ramsay, G., Cropper, M., \& Mason, K. O. 1996, MNRAS, 278, 285

Ratering, C., Bruch, A., \& Diaz, M. 1993, A\&A, 268, 694

Robertson, J. W., Howell, S. B., Honeycutt, R. K., Kafka, S., \& Campbell, T. 2008, AJ, 136, 1857

Schwope, A. D., Schwarz, R., \& Greiner, J. 1999, A\&A, 348, 861

Shakhovskoy, N. M., Alexeev, I. Y., Andronov, I. L., \& Kolesnikov, S. V. 1993, Cataclysmic Variables Relat. Phys., 10, 237

Spruit, H. C. 1998, arXiv:astro-ph/9806141

Thorstensen, J. R., Schommer, R. A., \& Charles, P. A. 1983, PASP, 95, 140

Visvanathan, N., Bessell, M. S., \& Wickramasinghe, D. T. 1984, IAU Circ., 3923, 2

Visvanathan, N., \& Pickles, A. 1982, Nature, 298, 41 
Walter, F. 2009, Wild Stars in the Old West. II. The 14th North American Workshop on Cataclysmic Variables and Related Objects (http://www.noao.edu/meetings/wildstars2/wildstars-presentations.php)

Watson, C. A., Dhillon, V. S., Rutten, R. G. M., \& Schwope, A. D. 2003 , MNRAS, 341, 129

Watson, C. A., Steeghs, D., Shahbaz, T., \& Dhillon, V. S. 2007, MNRAS, 382, 1105

Webbink, R. F., \& Wickramasinghe, D. T. 2002, MNRAS, 335, 1
Webbink, R. F., \& Wickramasinghe, D. T. 2005, in ASP Conf. Ser. 330 The Astrophysics of Cataclysmic Variables and Related Objects, ed. J.-M. Hameury \& J.-P. Lasota (San Francisco, CA: ASP), 137

Wickramasinghe, D. T., Visvanathan, N., \& Tuohy, I. R. 1984, ApJ, 286, 328

Wolff, M. T., Wood, K. S., Imamura, J. N., Middleditch, J., \& Steiman-Cameron, T. Y. 1999, ApJ, 526, 435

Wright, A. E., Stewart, R. T., Nelson, G. J., Slee, O. B., \& Cropper, M. 1988, MNRAS, 231, 319 